Europhysics Letters

PREPRINT

\title{
On the pearl size of hydrophobic polyelectrolytes
}

\author{
D. Baigl ${ }^{1}$, M. Sferrazza ${ }^{1,2}$ and C. E. $\operatorname{Williams}^{1}\left(^{*}\right)$ \\ 1 Laboratoire des Fluides Organisés, CNRS UMR 7125, Collège de France - 11, place \\ Marcelin Berthelot, 75005 Paris, France. \\ 2 Department of Physics, University of Surrey - Guildford, Surrey GU2 7XH, UK.
}

PACS. 82.35.Rs - Polyelectrolytes.

PACS. 81.07. Nb - Molecular nanostructures.

PACS. 68.47.Mn - Polymer surfaces.

\begin{abstract}
Hydrophobic polyelectrolytes have been predicted to adopt an unique pearlnecklace conformation in aqueous solvents. We present in this Letter an attempt to characterise quantitatively this conformation with a focus on $D_{p}$, the pearl size. For this purpose polystyrenesulfonate (PSS) of various effective charge fractions $f_{\text {eff }}$ and chain lengths $N$ has been adsorbed onto oppositely charged surfaces immersed in water in condition where the bulk structure is expected to persist in the adsorbed state. In situ ellipsometry has provided an apparent thickness $h_{\text {app }}$ of the PSS layer. In the presence of added salts, we have found: $h_{a p p} \sim a N^{0} f_{\text {eff }}^{-2 / 3}$ ( $a$ is the monomer size) in agreement with the scaling predictions for $D_{p}$ in the pearl-necklace model if one interprets $h_{a p p}$ as a measure of the pearl size. At the lowest charge fractions we have found $h_{a p p} \sim a N^{1 / 3}$ for the shorter chains, in agreement with a necklace/globule transition.
\end{abstract}

Introduction. - Polyelectrolytes are called hydrophobic when water is a poor solvent for the backbone. Because many artificial or natural macromolecules may have some intrinsic hydrophobicity, understanding the behaviour of hydrophobic polyelectrolytes constitutes a challenging area for fundamental and practical physical studies [1]. The most spectacular property of hydrophobic polyelectrolytes is the pearl-necklace conformation predicted for the single chain. This unusual conformation was obtained theoretically by Dobrynin et al. 2,3 by analogy with the Rayleigh instability of a charged droplet 4,5. Indeed a neutral hydrophobic polymer when immersed in water forms a spherical globule (like a drop of oil in water) in order to minimise the interfacial area with the solvent. When charges are added to the polymer the associated electrostatic energy of the globule increases until it balances the interfacial energy at some critical charge fraction $f_{C}$. Beyond $f_{C}$ the globule splits into two smaller ones thus increasing the interfacial area but decreasing the electrostatic energy. Contrary to the case of a charged droplet, the presence of connected monomers hinders infinite separation and an elongated string is formed between the pearls. Therefore the resulting structure is a water-soluble pearl-necklace composed of dense pearls connected by narrow strings (most of the monomers are contained in the pearls). Scaling theory provides the description of

$\left({ }^{*}\right)$ E-mail: claudine.williams@college-de-france.fr

(c) EDP Sciences 
hydrophobic polyelectrolytes properties [2, 3, which we summarise here. The pearl density $\rho$ is that of a globule of a neutral chain in a poor solvent $\rho \sim\left(a^{2} \xi_{\mathrm{T}}\right)^{-1}$ where $a$ is the monomer size and $\xi_{\mathrm{T}}$ is the thermal blob size. A pearl has a charge of $\rho D_{p}^{3} f_{\text {eff }}$ e where $f_{\text {eff }}$ is the effective linear charge density $\left(f_{e f f}=0.1\right.$ or $10 \%$ means that every tenth monomer is effectively charged in average) and e is the elementary charge. When the electrostatic energy balances the interfacial energy (proportional to $D_{p}^{2}$ ), we have:

$$
\frac{\left(\rho D_{p}^{3} f_{e f f} \mathrm{e}\right)^{2}}{\epsilon D_{p}} \sim k_{\mathrm{B}} T \frac{D_{p}^{2}}{\xi_{\mathrm{T}}^{2}}
$$

and the pearl size scales as:

$$
D_{p} \sim a\left(\frac{l_{\mathrm{B}}}{a}\right)^{-1 / 3} f_{e f f}^{-2 / 3}
$$

$l_{\mathrm{B}}$ is the Bjerrum length $\left(\sim \mathrm{e}^{2} /\left(\epsilon k_{\mathrm{B}} T\right)\right)$ and equals $0.7 \mathrm{~nm}$ in pure water at $25^{\circ} \mathrm{C}$. The distance between pearls, the string length $l_{\text {string }}$, results from the balance of repulsion between two neighbouring pearls with surface energy of the string, proportional to $l_{\text {string }}$ :

$$
\begin{gathered}
\frac{\left(\rho D_{p}^{3} f_{\text {eff }} \mathrm{e}\right)^{2}}{\epsilon l_{\text {string }}} \sim k_{\mathrm{B}} T \frac{l_{\text {string }}}{\xi_{\mathrm{T}}} \\
l_{\text {string }} \sim a\left(\frac{l_{\mathrm{B}} \xi_{\mathrm{T}}}{a^{2}}\right)^{-1 / 2} f_{\text {eff }}^{-1}
\end{gathered}
$$

Simulations also showed a pearl-necklace conformation [6] and more recently confirmed that pearl formation was due to an instability since pearls were observed to fluctuate along the chain in number and in size [7. Experimentally, Essafi et al. studied poly(styrene- $c o$-sodium styrenesulfonate), abbreviated PSS, as a model hydrophobic polyelectrolyte. By measuring the fluorescence emission of pyrene probes they observed the existence of low dielectric constant nano-regions dispersed along the chains [8] in agreement with the presence of hydrophobic pearls on the chains. They measured also the effective charge fraction as a function of the chemical charge fraction by osmometry [9. In a range of charge fraction where hydrophilic polyelectrolytes followed rather well the so-called Manning-Oosawa condensation theory 10 , [11, they found for PSS a further reduction of $f_{\text {eff }}$ according to the empirical linear law:

$$
f_{\text {eff }}(\%)=\frac{35(f(\%)-27)}{73}
$$

This effect is compatible with the pearl-necklace model if one also takes into account ionomer effects due to the low local dielectric constant in the pearls. Further experimental evidences for the pearl-necklace conformation may be found in [1] and references within.

However quantitative investigations of the single chain pearl-necklace conformation are difficult to perform experimentally on chains in solution. This is due in part to the low contrast in scattering experiments which prevents dilute solution measurement and also to the fluctuating nature of polyelectrolyte solutions. An interesting alternative consists in looking at chain conformation in the adsorbed state. Théodoly et al. showed that hydrophobic polyelectrolytes adsorbed spontaneously at a hydrophobic interface but adopted a flat conformation whatever charge fraction, the pearls unfolding on the surface. The film thickness was then independent of $f$ [12. For the investigation reported here hydrophobic polyelectrolytes have been adsorbed electrostatically onto oppositely charged solid surfaces in experimental conditions where the pearl-necklace conformation is expected to persist on the surface [13]. The thickness of the 
TABLE I - PSS samples of various chain lengths $N$, chemical charge fractions $f$ and effective charge fractions $f_{\text {eff }}$. $f_{\text {eff }}$ is calculated from $f$ according to the empirical renormalisation law (eq. 5)

\begin{tabular}{lllcccrrr}
$N$ & $f(\%)$ & $f_{\text {eff }}(\%)$ & $N$ & $f(\%)$ & $f_{\text {eff }}(\%)$ & $N$ & $f(\%)$ & $f_{\text {eff }}(\%)$ \\
120 & 34 & 3.4 & 930 & 33 & 2.9 & 1320 & 36 & 4.3 \\
120 & 54 & 13 & 930 & 41 & 6.7 & 1320 & 53 & 12 \\
120 & 64 & 18 & 930 & 46 & 9.1 & 1320 & 71 & 21 \\
120 & 94 & 32 & 930 & 53 & 12 & 1320 & 91 & 31 \\
\hline 410 & 39 & 5.8 & 930 & 64 & 18 & 1320 & 100 & 35 \\
410 & 56 & 14 & 930 & 83 & 27 & 2520 & 37 & 4.8 \\
410 & 71 & 21 & & & & 2520 & 54 & 13 \\
410 & 100 & 35 & & & & 2520 & 89 & 30
\end{tabular}

adsorbed monolayer has been measured by in situ ellipsometry and interpreted afterwards in terms of the pearl size $D_{p}$.

In this study we used also PSS as a model hydrophobic polyelectrolyte. Control of the chemistry [14] allowed us to synthesise and characterise a series of well defined monodisperse $\left(M_{W} / M_{N}<1.3\right)$ hydrophobic polyelectrolytes of various chain lengths and charge fractions (linear charge densities) as summarised in Table \. Fully charged PSS's $(f=100 \%)$ were purchased from Fluka.

PSS solutions were prepared at a polymer concentration of $0.01 \mathrm{~mol} / \mathrm{L}$ in deionised water (Millipore water) with added salt $(\mathrm{NaCl})$ or in salt-free conditions. Positively charged surfaces were prepared by grafting on silicon wafers a self assembled monolayer (SAM) with terminal amino groups composing the upper surface and obtained by the reaction of (3-Aminopropyl)trimethoxysilane on the silica covering the wafer and previously activated. Silica activation consisted of 45 minutes $\mathrm{UV}-\mathrm{O}_{3}$ treatment prior to 15 minutes exposure under a $\mathrm{O}_{2}+$ $\mathrm{H}_{2} \mathrm{O}$ gas flow. Silanation was made in liquid phase (toluene). The SAM quality (thickness $\simeq 1.1 \mathrm{~nm}$ and roughness of the bare silicon) was controlled in air by ellipsometry and Xray reflectivity. In situ ellipsometry measurements were performed using a variable angle spectroscopic ellipsometer (VASE Woollam Inc., USA) with a rotating analyzer configuration. The sample was contained in a liquid cell with thin glass windows perpendicular to the incident light beam at $72^{\circ}$ with respect to the sample normal. The ellipsometric angles $\Psi$ and $\Delta$ were measured as a function of the wavelength $\lambda(400 \mathrm{~nm} \leq \lambda \leq 700 \mathrm{~nm})$. A three layer $\left(\mathrm{SiO}_{2} / \mathrm{SAM} / \mathrm{PSS}\right)$ optical model was used for the fitting of the ellipsometric angles. The thicknesses of the first two layers were kept to the previously measured values: $\mathrm{SiO}_{2}$ in air prior to SAM grafting, SAM in water before PSS adsorption. The PSS layer has been modelled with an uniform refractive index $n_{\text {PSSlayer }}$ and an apparent thickness $h_{\text {app }}$.

First of all it was found that, whatever chain length, charge fraction or ionic strength conditions, the adsorption of PSS onto oppositely charged surfaces presented some general features: the adsorption was very fast and equilibrium was reached after a few minutes; at equilibrium several consecutive measurements were performed showing that the layer was stable; when the adsorbing solution was flushed by a $\mathrm{NaCl}$ solution at the same ionic strength (or pure water in case of salt-free adsorption) the layer thickness remained constant; when this $\mathrm{NaCl}$ solution was flushed by deionised water there was no measurable change. This shows the strongly irreversible character of this electrostatically driven adsorption. In general the layer characteristics only depended on the bulk conditions (ionic strength) during the adsorption process. 


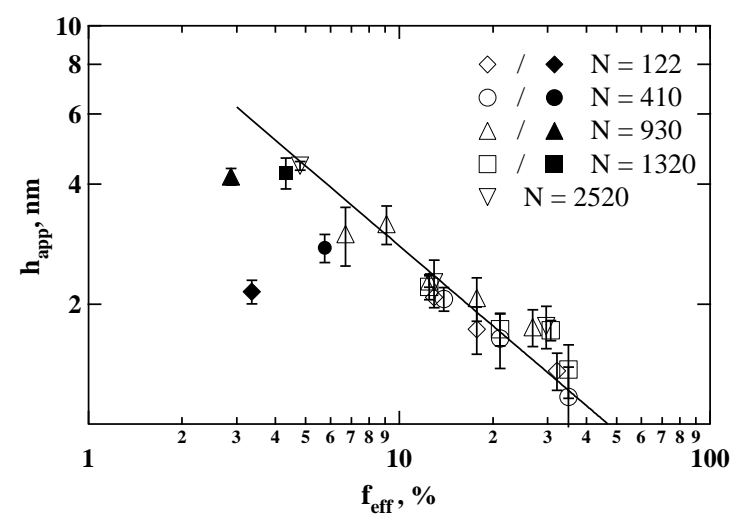

Fig. 1 - Apparent thickness $h_{a p p}$ of adsorbed layers as a function of the effective charge fraction $f_{\text {eff }}$ for PSS of various chain lengths. Each point is an average of 12 measurements. For this adsorbing conditions $\left(C_{p}=0.01 \mathrm{~mol} / \mathrm{L},[\mathrm{NaCl}]=0.1 \mathrm{~mol} / \mathrm{L}\right)$, this thickness can be interpreted as the pearl size of PSS. The straight line has a slope of $-2 / 3$. Filled points are reported in fig. 2

First, PSS's were adsorbed from salted solutions $([\mathrm{NaCl}]=0.1 \mathrm{~mol} / \mathrm{L})$. The ionic strength was chosen so that the Debye length $\lambda_{D}$ was so small $\left(\lambda_{D}<1 \mathrm{~nm}\right)$ that pearls could come very close without any significant repulsion but large enough to allow PSS adsorption. If, as theory predicts, the bulk conformation remains in the adsorbed state, the adsorbed layer could be imagined as a dense carpet of pearls.

The ellipsometric determination of $h_{\text {app }}$ requires the knowledge of the refractive index of the adsorbed layer $n_{\text {layer }}$. Fits of the ellipsometric angles gave for all samples a value of $n_{\text {layer }} \simeq 1.45$. This value is compatible with that estimated for a compact layer of pearls (remember that the majority of PSS monomers belong to the dense pearls). Using a linear approximation $n_{\text {layer }}$ is the average between that of dry PSS (1.53 [15]) and that of water (1.33) weighted by the respective volume fractions $\left(\Phi_{\text {pearl }}=\pi /(3 \sqrt{3}) \simeq 0.60\right.$ for a compact hexagonal monolayer) and one finds also a value of 1.45. Therefore, in what follows, the layer index has been fixed to $n_{\text {layer }}=1.45$ and $\Psi$ and $\Delta$ interpreted in terms of the apparent thickness $h_{\text {app }}$.

The dependence of $h_{a p p}$ on the effective charge fraction $f_{\text {eff }}$ is presented in fig. 1 for various chain lengths $N$. It shows clearly that $h_{a p p}$ depends significantly on the effective charge fraction whereas it is independent of $N$ (if one ignores the filled symbols which will be considered later). Within experimental errors $h_{\text {app }}$ obeys the following power law:

$$
h_{a p p} \sim a N^{0} f_{e f f}^{-2 / 3}
$$

In order to interpret this charge density dependence we assume that $h_{a p p}$ is closely related to the pearl size $D_{p}$ (PSS layer viewed as a carpet of pearls as noted above). The $N$ and $f$ exponents are indeed in remarkable agreement with that for $D_{p}$ predicted for the bulk conformation (eq. 21) in the pearl-necklace model. This confirms that we are in the conditions where the pearl-necklace conformation persists upon electrostatic adsorption as it has been predicted theoretically in the case of highly charged hydrophobic polyelectrolytes [13]. Thus we identify the $N$ and $f$ exponents found here to those for $D_{p}$.

Let us now come back to the filled points in fig. 1 that deviate from the power law (eq. 6) and correspond to the lowest $N$ and $f$ values. They are reported in fig. 2 where $h_{\text {app }}$ for these 


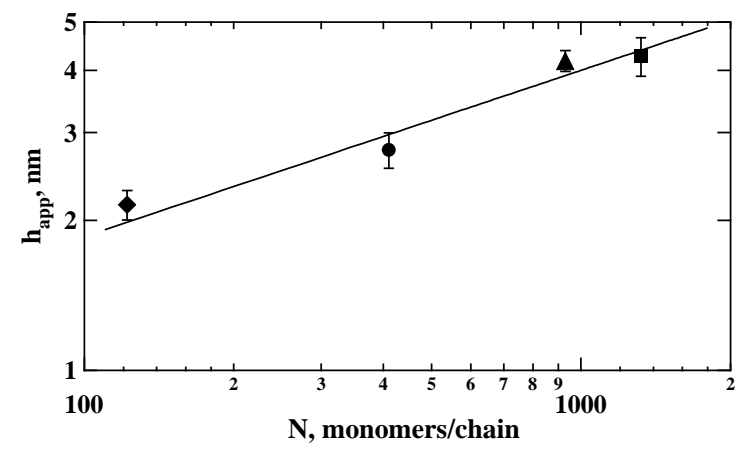

Fig. 2 - Apparent thickness $h_{a p p}$ of adsorbed layers versus chain length $N$ for short PSS $(120 \leq N \leq$ $1320)$ at very low charge fractions $\left(f_{e f f}<5 \%\right)$. The points correspond to filled points in fig. 1] The straight line has a slope of $1 / 3$.

points is plotted as a function of the chain length. Experimentally $h_{a p p}$ scales as

$$
h_{a p p} \sim a N^{1 / 3}
$$

Because in this case $f_{e f f}$ is low the theoretically predicted pearl size becomes very large and can even be larger than what the number of monomers allows. This means than for these points (low $N$, low $f_{e f f}$ ), the chain is not envisioned as a pearl-necklace with many pearls at equilibrium but instead as a dense and spherical globule minimising its interfacial area with the solvent. The globule size is less than $D_{p}$ and is stable because its electrostatic energy is less than its interfacial energy. Similarly to pearls, globules are expected to be close compacted because of the very short-range repulsion. We thus expect that $h_{\text {app }}$ is here proportional to the globule size. The $1 / 3$ exponent as represented by the straight line agrees indeed with a compact globular structure of the collapsed chain. This is an indirect evidence of a pearl-necklace/globule transition that is controlled ar low $f$ by the chain length $N$.

It has to be pointed out that ellipsometry does not allow one to characterise the shape of a single pearl or globule. Nevertheless the identity of the scaling exponents indicate that only weak deformations of the pearls (globules respectively) would take place. This is to be expected with our experimental conditions of strong screening of the electrostatic attraction to the surface $\left(\lambda_{D}<D_{p}\right)$. Furthermore we believe that dipolar attractive forces within the pearls (ionomer effect) stabilise the conformation [16].

It is interesting to contrast these properties to that of layers adsorbed from salt-free solutions. PSS of same chain lengths and charge fractions were adsorbed from salt-free solutions at $C_{p}=0.01 \mathrm{~mol} / \mathrm{L}$. Because the free counter-ion concentration $\left(\simeq C_{p} f_{\text {eff }}\right)$ is low, the Debye length $\lambda_{D}$ is very large $(10$ to $30 \mathrm{~nm})$ and one expects that the pearl-necklace adopts a stretched conformation in the bulk. For a direct comparison with the previous results the refractive index is arbitrarily kept at $n_{\text {PSSlayer }}=1.45$, the refractive index of the dense carpet of pearls. The resulting apparent thickness $h_{a p p}$ is plotted in fig. [3 depending on $f_{\text {eff }}$ for PSS of various chain lengths. Within experimental errors $h_{a p p}$ is found independent of both $f_{\text {eff }}$ and $N$. Using the same $n_{\text {layer }}$ the film appears thin and the thickness is smaller than the unperturbed pearl size. Two limit interpretations can be envisioned. The first one assumes that the free chain conformation remains in the adsorbed state. In this case pearls still exist but they are separated by extended string immersed in water. The apparent thickness appears constant because the string length, the distance between two neighbouring pearls, increases 
EUROPHYSICS LETTERS

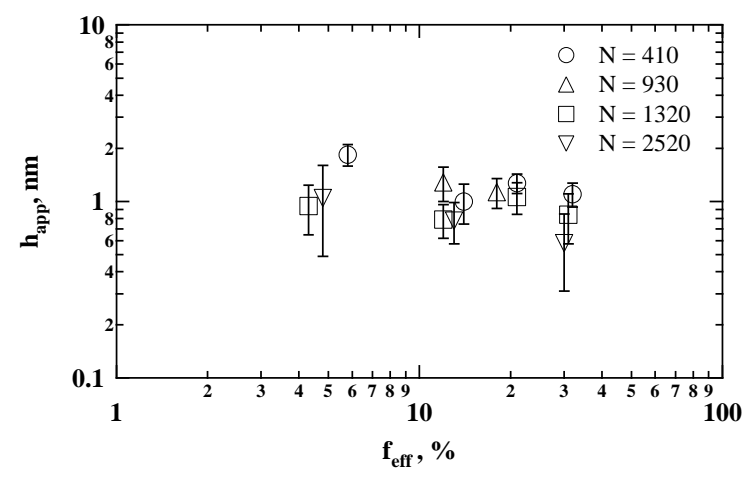

Fig. 3 - Apparent thickness $h_{a p p}$ versus effective charge fraction $f_{\text {eff }}$ for PSS of various chain lengths $N$ adsorbed from salt-free solutions.

when the pearl size increases. The second one takes into account the fact that the electrostatic attraction onto the oppositely charged surface is much stronger than that in the salted regime $\left(\lambda_{D} \gg D_{p}\right)$ and assumes that pearls spread on the surface. A smooth monolayer is formed with a thickness of the order of the thermal blob size $\xi_{T}$ which does not depend on $f$ similarly to what has predicted a recent theoretical model [17. In situ ellipsometry is not capable to differentiate these two scenarii.

Conclusion. - A model hydrophobic polyelectrolyte, partially sulfonated polystyrenesulfonate (PSS) of various chain lengths $N$ and effective charge fractions $f_{\text {ef } f}$, has been adsorbed onto oppositely charged surfaces in conditions where the pearl-necklace conformation predicted for the single chain in the bulk is expected to be maintained in the adsorbed state. The apparent layer thickness $h_{a p p}$ has been measured by in situ spectroscopic ellipsometry as a function of $f_{\text {eff }}$ and $N$ and we have found $h_{a p p} \sim a N^{0} f_{e f f}^{-2 / 3}$. If we assume that $h_{a p p}$ is proportional to the pearl size, this would be the first experimental confirmation of the predicted scaling law (eq. 2) relating the pearl size to the charge fraction in the pearl-necklace model 2]. At low $f_{\text {eff }}$ we have found $h_{a p p} \sim a N^{1 / 3}$ for the shortest chains. We interpret this power law as an evidence of a globule/necklace transition at low $f$ controlled by $N$. Moreover it is interesting to note that we have preliminary results from in situ high energy X-ray reflectivity measurements [18] that confirm the scaling law for $h_{a p p}$. Fig. 4 displays the PSS thickness obtained by this technique which indeed depends on the effective charge fraction with the same $-2 / 3$ scaling exponent. On the other hand an important feature is that the scaling laws reported here have been expressed in terms of $f_{\text {eff }}$ rather than $f$, the bare charge. This shows that $f_{\text {eff }}$ is the experimental parameter to take into account for a correct estimation of the electrostatic contribution. Further experiments will explore the influence of the surface charge density and in situ AFM in soft contact mode will allow us to image the adsorbed PSS molecules.

We wish to acknowledge J. L. Keddie and C. Carelli (University of Surrey) for their helpful support during the in situ ellipsometry measurements. 


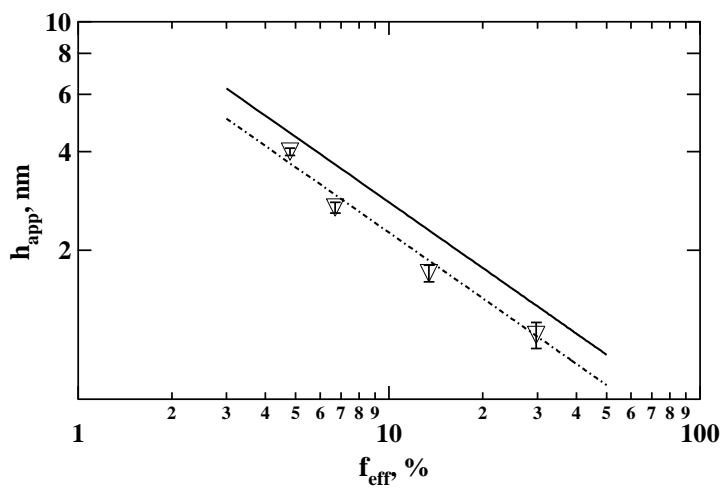

Fig. 4 - Apparent thickness $h_{a p p}$ as a function of the effective charge fraction $f_{\text {eff }}$ as measured by high energy in situ X-ray reflectivity. The continuous line is that of fig. 1 The dashed line has also a slope of $-2 / 3$. Measurements were performed in a custom made liquid cell using high energy (19 keV) X-ray from the ESRF synchrotron (Grenoble, France) and will be detailed in a forthcoming paper 18.

\section{REFERENCES}

[1] Williams C. E., Electrostatic Effects in Soft Matter and Biophysics, edited by C. Holm, P. KÉKicheff and R. Podgornik (Kluwer Academic Publishers, Dordrecht) 2001, p. 487

[2] Dobrynin A. V., Rubinstein M. and Obukhov S. P., Macromolecules, 29 (1996) 2974

[3] Dobrynin A. V. and Rubinstein M., Macromolecules, 32 (1999) 915

[4] Lord Rayleigh, Philos. Mag., 14 (1882) 184

[5] The necklace conformation by analogy with the Rayleigh instability of a charged droplet was first introduced by Kantor and Kardar in the case of polyampholytes: KANTOR Y. and KARDAR M., Europhys. Lett., 27 (1994) 643

[6] Micka U., Holm C. and Kremer K., Langmuir, 15 (1999) 4033

[7] Limbach H.-J., Holm C. and Kremer K., Europhys. Lett., 60 (2002) 566

[8] Essafi W., Lafuma F. and Williams C. E., J. Phys. II, 5 (1995) 1269

[9] Essafi W., PhD Thesis, Paris VI (1996)

[10] Manning G. S., J. Chem. Phys., 51 (1969) 924

[11] Oosawa F., Polyelectrolytes, Vol. 9 (Marcel Dekker, New-York) 1971

[12] Théodoly O., Ober R. and Williams C. E., Eur. Phys. J. E., 5 (2001) 51

[13] Dobrynin A. V. and Rubinstein M., Macromolecules, 35 (2002) 2754

[14] Baigl D., Seery T. A. P. and Williams C. E., Macromolecules, 35 (2002) 2318

[15] ThÉOdoly O., PhD Thesis, Paris VI (1999)

[16] Experimentally it is known that the dielectric constant within the pearls is low enough to produce undissociated ion-pairs [8]. The subsequent dipolar attraction of these into multiplets leads to the formation of physical crosslinks which could stabilise the pearl conformation. The same ionomer effect is invoked to describe the super-collapsed state of polyelectrolyte gels: KHOKHLOV A. R. and Kramarenko E. Yu.,Macromolecules, 29 (1996) 681

[17] Borisov O. V., Hakem F., Vilgis T. A., Johnny J.-F. and Johner A., Eur. Phys. J. E, 6 (2001) 37

[18] Baigl D., Ober R., Sferrazza M., Boesecke P., Jean B. and Williams C. E., In preparation 